\title{
A numerical study of flow around an impulsively started circular cylinder by a deterministic vortex method
}

\author{
By CHIEN-CHENG CHANG AND RUEY-LING CHERN \\ Institute of Applied Mechanics, College of Engineering, National Taiwan University, \\ Taipei 10764, Taiwan, R.O.C.
}

(Received 10 April 1989 and in revised form 4 April 1991)

Impulsively started flow around a circular cylinder at various Reynolds numbers is studied by a deterministic hybrid vortex method. The key feature of the method consists in solving the viscous vorticity equation by interlacing a finite-difference method for diffusion and a vortex-in-cell method for convection. The vorticity is updated along the surface of the cylinder to satisfy the no-slip condition. The present method is basically different from previous applications of vortex methods, which are primarily in the context of random vortex algorithms. The Reynolds numbers of the flows under investigation range from 300 to $10^{6}$. Numerical results are compared with analytical solutions at small times, and compared with finite-difference solutions and flow visualization results at relatively long times. Satisfactory agreement is found in the evolutions of the separation angles, wake lengths, surface pressure and drag coefficients, streamline patterns, and some velocities on the axis of symmetry behind the circular cylinder. The present hybrid vortex method is highly stable and suffers from little numerical diffusivity, yielding convincing numerical results for unsteady vortical flows at moderately high Reynolds numbers.

\section{Introduction}

There has been a continuing interest in capturing complex structures of flows around an impulsively started circular cylinder. Considerable effort has been directed to finding analytical solutions (see e.g. Wang 1967; Collins \& Dennis 1973a; and BarLev \& Yang 1975) and accurate finite-difference solutions (see e.g. Thoman \& Szewczyk 1969; Collins \& Dennis 1973 $b$; Ta Phuoc Loc 1980 among others). However, all the previously known analytical solutions are only valid at very small times, while finite-difference methods often suffer from numerical instability or/and numerical diffusivity. Therefore for flows at high Reynolds numbers most traditional numerical methods may not be adequate for simulation, especially in long-time calculations.

To overcome the numerical difficulties, heuristic vortex methods using random walks for modelling viscous diffusion and an inviscid vortex method for handling convection were proposed by Chorin $(1973,1978)$ in the study of slightly viscous flows. The methods have thereafter received much attention in the simulation of unsteady vortical flows, especially at high Reynolds numbers; see, for example, Cheer (1983, 1989), Ghoniem, Chorin \& Oppenheim (1982), Sethian (1984), Tiemroth (1986) and Smith \& Stansby (1988). In the authors' opinion, although the random vortex method may have great value in simulating fully developed turbulent flows, 

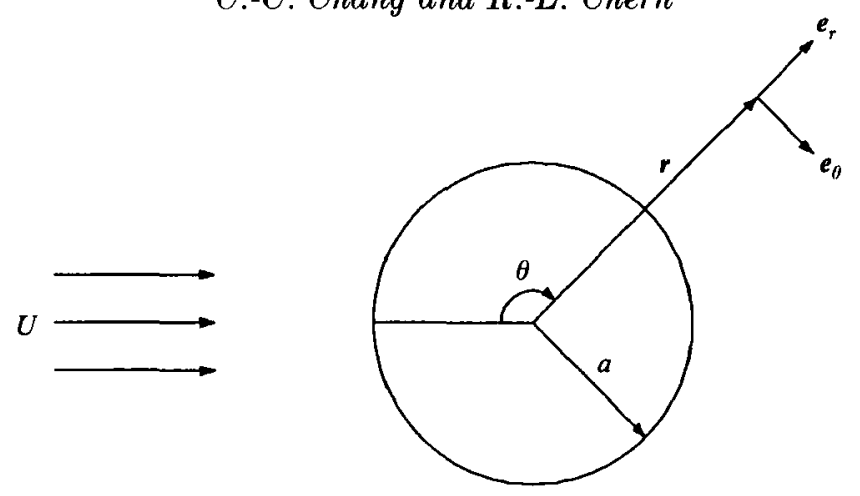

Figure 1. Schematic of the physical problem.

it is not very satisfactory for use in studying problems of transition and stability. Typically, one has to resort to smoothing techniques to obtain good quantitative agreement between computed results and analytical or finite-difference results in employing a random approach. We refer to Leonard $(1980,1985)$ and Sarpkaya (1989) for a detailed review of computational methods with vortices for viscous and non-viscous flows.

Motivated by the above observations, we present in this article a deterministic vortex algorithm without using random walks to study the impulsively started flow around a circular cylinder, which is shown to be highly stable during a long period of calculation. Roughly speaking, the method is of fractional-step type: diffusion is solved by finite difference and convection is solved by a vortex-in-cell method. The present method avoids therefore the use of random walks and difficulties followed from it whilst remaining highly stable and practically free from the problem of numerical diffusivity. However, the vortex-in-cell procedure requires a mesh in the computational domain. Following Smith \& Stansby (1988) we adopt a single, radially expanding polar mesh, the inner side of which coincides with the surface of the cylinder along which the vorticity is updated to satisfy the no-slip condition. The mesh is also used in the present study to solve the diffusion equation in order to take into account the effect of viscous diffusion.

The flow around an impulsively started circular cylinder (cf. figure 1) is known to become eventually three-dimensional when the Reynolds number is increased above about 200 (cf. Tritton 1988, p. 30). However, careful flow visualization reveals that the flow in the early stage of development in the (laminar) wake region is nearly twodimensional; the typical phenomena include a gross change of wake region and the formation of primary and secondary vortices. The formation of secondary vortices is of special interest; their appearance may soon affect the flow structure and other flow properties. As shown in figure 2, a single secondary vortex may result from a bulge phenomenon, and form an isolated eddy, while there may also be secondary vortices forming the $\alpha$ - or $\beta$-(forewake) phenomenon as classified by Bouard \& Coutanceau (1980). This study concerns flows at moderately high Reynolds numbers in the range from 300 to $10^{6}$. Numerical results are presented for time variations of the separation angles, wake lengths, surface pressure and vorticity distributions, drag coefficients, streamline patterns and some velocities on the symmetric axis behind the cylinder. Previous numerical results show reasonable agreement with analytical solutions at small times and with flow visualization experiments at relatively long times. These may serve as a check of validity of the present hybrid vortex method. The analytical solution used for comparison is that of Collins \& Dennis (1973a); finite-difference 
(a)

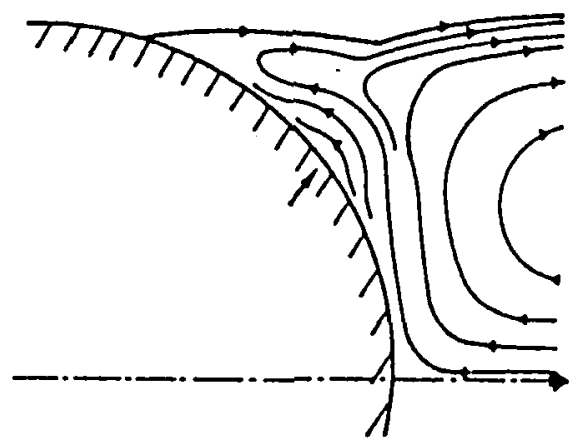

(c)

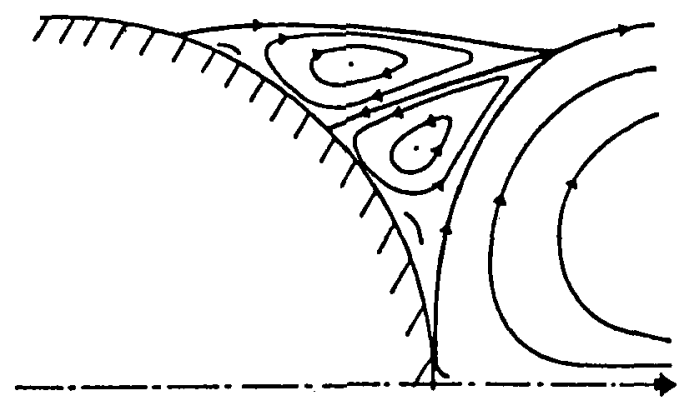

(b)

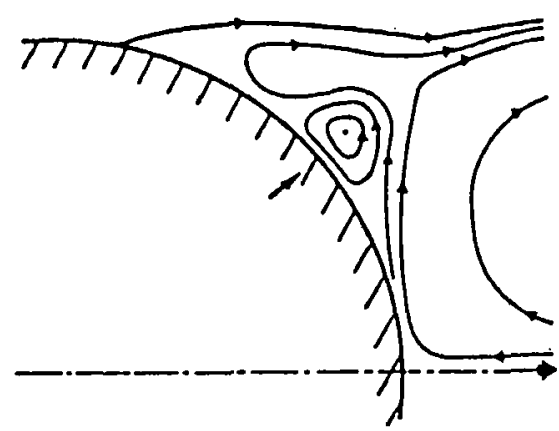

(d)

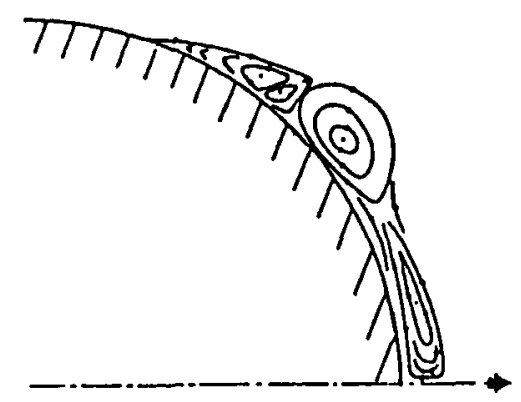

FIGURE 2. Flow patterns near the surface of the cylinder: $(a)$ bulge phenomenon, $(b)$ isolated secondary vortex, (c) $\alpha$-phenomenon, (d) $\beta$-phenomenon.

solutions for this purpose are taken from Ta Phuoc Loc (1980) and Ta Phuoc Loc \& Bouard (1985), while the work of Bouard \& Coutanceau (1980) serves as an experimental check. All the comparisons show generally close agreement; somewhat different results are, however, observed at relatively high Reynolds numbers. This discrepancy with experimental results occurs earlier for flows at higher Reynolds numbers for which the real flow hardly remains two-dimensional and becomes turbulent eventually. The discrepancy with the finite-difference solutions may be due to the numerical diffusivity associated with the finite-difference method, the effect of which increases with time. Nevertheless, we observe that there is a consistent trend that the bulge phenomenon is typical prior to the formation of a substantial isolated secondary vortex. The $\alpha$-phenomenon is typically observed at high Reynolds numbers, and the $\beta$-phenomenon usually appears in the early phase of flow establishment, also at high Reynolds numbers. These can be seen from both the streamline patterns and the vorticity distribution over the surface of the circular cylinder.

\section{Basic equations}

We follow the formulation of the problem described by Smith \& Stansby (1988). Consider a fluid of density $\rho$ and constant kinematic viscosity $\nu$. The flow past a circular cylinder is governed by the Navier-Stokes and the continuity equations. 
Some flow parameters are shown in figure 1. Let the reference length and velocity be the cylinder radius $(a)$ and the speed of the distant flow $(U)$ respectively. In dimensionless form, these equations may be written

$$
\begin{gathered}
\partial u / \partial t+(\boldsymbol{u} \cdot \nabla) \boldsymbol{u}=-\nabla P+2 / \operatorname{Re} \nabla^{2} \boldsymbol{u}, \\
\nabla \cdot u=0,
\end{gathered}
$$

where $u$ denotes the velocity and $P$ the pressure, and $R e=2 U a / \nu$ is the Reynolds number. The dimensional velocity $u^{*}$, time $t^{*}$ and pressure $P^{*}$ are given by

$$
u^{*}=U u, \quad t^{*}=a t / U, \quad P^{*}=\rho U^{2} P .
$$

For the present study, it is convenient to work with a stream function-vorticity formulation. By introducing an appropriate stream function and the vorticity function $\omega=\nabla \times u$, we may recast equations (1) and (2) as

and

$$
\begin{gathered}
\nabla^{2} \psi=-\omega \\
\partial \omega / \partial t+(u \cdot \nabla) \omega=2 / \operatorname{Re} \nabla^{2} \omega,
\end{gathered}
$$

where $\boldsymbol{\omega}=\omega \boldsymbol{k}$. Refer the motion to a polar coordinate system $(r, \theta)$ : the cylinder surface is at $r=1$ and the coordinate $(1,0)$ coincides with the front stagnation point of the corresponding potential flow.

Let $t=0$ define the start of the motion, the initial condition at $t=0+$ consists of the potential flow and a vorticity sheet at the cylinder surface. The boundary conditions for (4) are that, for $t>0$,

$$
\begin{gathered}
\psi=0 \text { for } r=1 \\
-\partial \psi / \partial r \rightarrow \sin \theta \quad \text { as } r \rightarrow \infty
\end{gathered}
$$

while the boundary conditions for (5) are that, for $t>0$,

$$
\begin{gathered}
\boldsymbol{u} \cdot \boldsymbol{e}_{\boldsymbol{\theta}}=\mathbf{0} \text { for } r=1, \\
\omega \rightarrow 0 \text { as } r \rightarrow \infty .
\end{gathered}
$$

The velocity $\boldsymbol{u}$ is related to the stream function $\psi$ through

$$
\boldsymbol{u}=\boldsymbol{e}_{r} / r \partial \psi / \partial \theta-\boldsymbol{e}_{\theta} \partial \psi / \partial r
$$

where $e_{r}$ and $e_{\theta}$ are the unit vectors along the radial and azimuthal directions respectively.

Let $D^{*}$ denote the drag exerted on the cylinder per unit length. The drag coefficient is defined by $C_{D}=D^{*} / \rho U^{2} a$ and can also be written as $C_{D}=C_{D P}+C_{D F}$ where $C_{D P}$ is due to pressure while $C_{D F}$ is due to friction. Let $P_{0}$ and $\omega_{0}$ denote the surface pressure and the surface vorticity respectively. The drag coefficient is then given by

$$
C_{D}=2 \int_{0}^{\pi} P_{0} \cos \theta \mathrm{d} \theta+\frac{4}{R e} \int_{0}^{\pi} \omega_{0} \sin \theta \mathrm{d} \theta .
$$

The surface pressure $P_{0}$ will be obtained, for each $\theta$, by integrating the momentum equation radially from infinity to the cylinder surface. Prescribing the value of $P$ to be 0 at infinity, we have

$$
\boldsymbol{P}_{0}(\theta)=\int_{1}^{\infty}\left(\frac{2}{R e} \frac{1}{r} \frac{\partial \omega}{\partial \theta}+\frac{\partial u_{r}}{\partial t}-u_{\theta} \omega\right) \mathrm{d} r+\frac{1}{2} .
$$




\section{The hybrid vortex method}

The present vortex method for incompressible viscous flow can be summarized as follows. The vorticity field is approximated by a sum of 'blob' functions - called vortex blobs or simply vortices. Each vortex evolves in a Lagrangian manner, carrying with itself a circulation determined from the vorticity. The vorticity is obtained by solving the vorticity diffusion equation on a grid by finite difference. The circulations are then redistributed and converted back to the vorticity on the grid, which is thus updated.

At the beginning of each time step there are only vortices centred at mesh points. Therefore, we first approximate the vorticity field by

$$
\omega(t, r)=\sum_{j=1}^{L} \Gamma_{j} f_{j}\left(r-r_{j}\right)
$$

where $L$ is the number of vortices (or mesh points) and $f_{j}(r)$ is the blob function. $\Gamma_{j}$ and $r_{j}$ are respectively the circulation and position associated with the $j$ th vortex blob. In practice, the function $f_{j}(r)$ is conveniently taken to be an indicator function so that the vorticity is constant over the chosen indicator set, outside which the blob function is zero. Highly accurate blob functions may be available in the form of combinations of some elementary functions; see, for example, Hald (1979), Beale \& Majda (1985).

Next we describe the polar coordinate system $\left(r^{\prime}, \theta\right)$ adopted by Smith \& Stansby in their work on random vortex contents. This was introduced for the solution of the Poisson equation (4) and for the creation of vorticity along the cylinder surface. For the present purpose, the coordinate system is also used to solve the vorticity diffusion equation in order to take into account the effect of viscous diffusion. In terms of the polar coordinates $\left(r^{\prime}, \theta\right)$ the Poisson equation (4) may be written

where

$$
\begin{gathered}
\partial^{2} \psi / \partial \theta^{2}+a\left(r^{\prime}\right) \partial^{2} \psi / \partial r^{2}+b\left(r^{\prime}\right) \partial \psi / \partial r^{\prime}=-\omega r^{2} \\
a\left(r^{\prime}\right)=\left(r \mathrm{~d} r^{\prime} / \mathrm{d} r\right)^{2}, \quad b\left(r^{\prime}\right)=r \mathrm{~d} r^{\prime} / \mathrm{d} r+r^{2} \mathrm{~d}^{2} r^{\prime} / \mathrm{d} r^{2} \\
r \equiv c\left(r^{\prime}\right)=B\left(\mathrm{e}^{A r^{\prime}}-1\right)+1 .
\end{gathered}
$$

For each time increment, $(12)$ is solved on a mesh with a uniform mesh size in the coordinate system $\left(r^{\prime}, \theta\right)$, defined over an annular region outside the cylinder surface $\left(1<r<r_{0}\right)$. Fine resolution is required near the surface of the cylinder. The constants $A, B$ in $(13 c)$ are fixed by the radial mesh spacing at the cylinder surface, and by the value of the outer radius $\left(r_{0}\right)$. The value of $r_{0}$ must be sufficiently large for $(7 b)$ to be an adequate approximation and for all the vortices to be contained within the mesh.

Denote by $(i, j)$ the node of the mesh; $r^{\prime}=j$ and $\theta=i \Delta \theta(\Delta \theta=2 \pi / p, 0 \leqslant j \leqslant q-1)$. Let $\psi(i, j)$ be the nodal value of the stream function at $(i, j)$. In a computation, (12) is solved by central finite difference in $r^{\prime}$ with the help of Fourier collocation for the second derivative in $\theta$. This yields a set of $p$ tridiagonal equations in $r^{\prime}$, which can be solved efficiently by Gaussian elimination. Once (12) is solved, the stream function is used to update the vorticity on the cylinder surface so that the no-slip condition is satisfied. Notice that along the cylinder surface, (12) reduces to

$$
\left.a\left(r^{\prime}\right) \frac{\partial^{2} \psi}{\partial r^{\prime 2}}\right|_{r^{\prime}=0}=-\omega(i, 0)
$$

Imagine that the stream function can be extended across the cylinder surface to possess meaningful values $\psi(i,-1)$. Then at least to a first-order approximation we 
require $\psi(i,-1)=\psi(i, 1)$ so that the tangential velocity along the cylinder surface is zero. Applying the central finite difference to (12) then yields the surface vorticity

$$
\omega(i, 0)=-2 a(0) \psi(i, 1)
$$

The value of $\omega(i, 0)$ in (14) can be evaluated accurately by interpolating the stream function using three or more radial mesh values near to the surface of the cylinder, followed by differentiation. With the solution of (12) and the surface vorticity $\omega(i, 0)$ in (15), the next step is to solve the diffusion equation on the grid by a finitedifference method.

$$
\partial \omega / \partial t=2 / R e \nabla^{2} \omega
$$

From the solution we construct the circulations to be convected by the vortices on the grid. Let $A_{i j}$ be the cell determined by $\left(i-\frac{1}{2}, j\right),\left(i+\frac{1}{2}, j\right),\left(i, j-\frac{1}{2}\right)$ and $\left(i, j+\frac{1}{2}\right)$. Each $A_{i j}$ corresponds to an indicator set in the original $r$-space. The circulation associated with the vortex at $(i, j)$ is then determined approximately by

$$
\Gamma(i, j)=\left.\omega(i, j) r \Delta \theta \frac{\mathrm{d} r}{\mathrm{~d} r^{\prime}}\right|_{r^{\prime}=j} .
$$

The formula is obtained by applying the midpoint rule for evaluating, in the $\left(r^{\prime}, \theta\right)$ plane, the integral of the vorticity over the area corresponding to $A_{i j}$. The vortex carrying $\Gamma(i, j)$ is convected to a new location with the velocity determined from (8). The circulation $\Gamma(i, j)$ is then redistributed between the four corner nodes of the cell in which the vortex is contained according to the area-weighting scheme suggested by Christiansen (1973). The reassignment of circulations completes a cycle of computation. The vortices moving away from the grid points are then discarded, while a new set of vortices are regenerated on the grid. Recall that the conversion of circulation into vorticity, and vice versa, is made through (17). The present hybrid vortex method therefore, for each time increment, consists of the following solution steps :

(i) Equation (12) for the stream function is solved on the grid; the solution is used to update the vorticity along the cylinder surface to satisfy the no-slip condition.

(ii) Equation (16) is solved on the grid by a finite-difference method; the circulation associated with each vortex on the grid is then evaluated according to (17).

(iii) Each vortex on the grid along with the associated circulation is convected with the velocity determined by (8) using the values of the stream function obtained in (i).

(iv) Redistribute all the circulations; add at each grid point the assigned circulations and convert the result into vorticity, again, according to (17). The current vortices are discarded while a new set of vortices are regenerated on the grid. Go back to (i).

The method described above is obviously of fractional-step type. The time accuracy can be improved by adopting a midpoint rule for evaluating the velocities of the vortices on the grid. One way of achieving this is to calculate the velocities using the average of the current vorticity and a predicted vorticity (both on the grid) at the next time step. This modified procedure is conceived to have second-order accuracy in time, and is used in the computations in the present study. Step (ii) takes care of the effect of diffusion while (iii) takes care of the convection. In the present approach vortices on the cylinder surface are not convected into the flow, while the diffusion of vorticity is achieved through solving the diffusion equation. The method is therefore completely deterministic, and is basically different from those using 


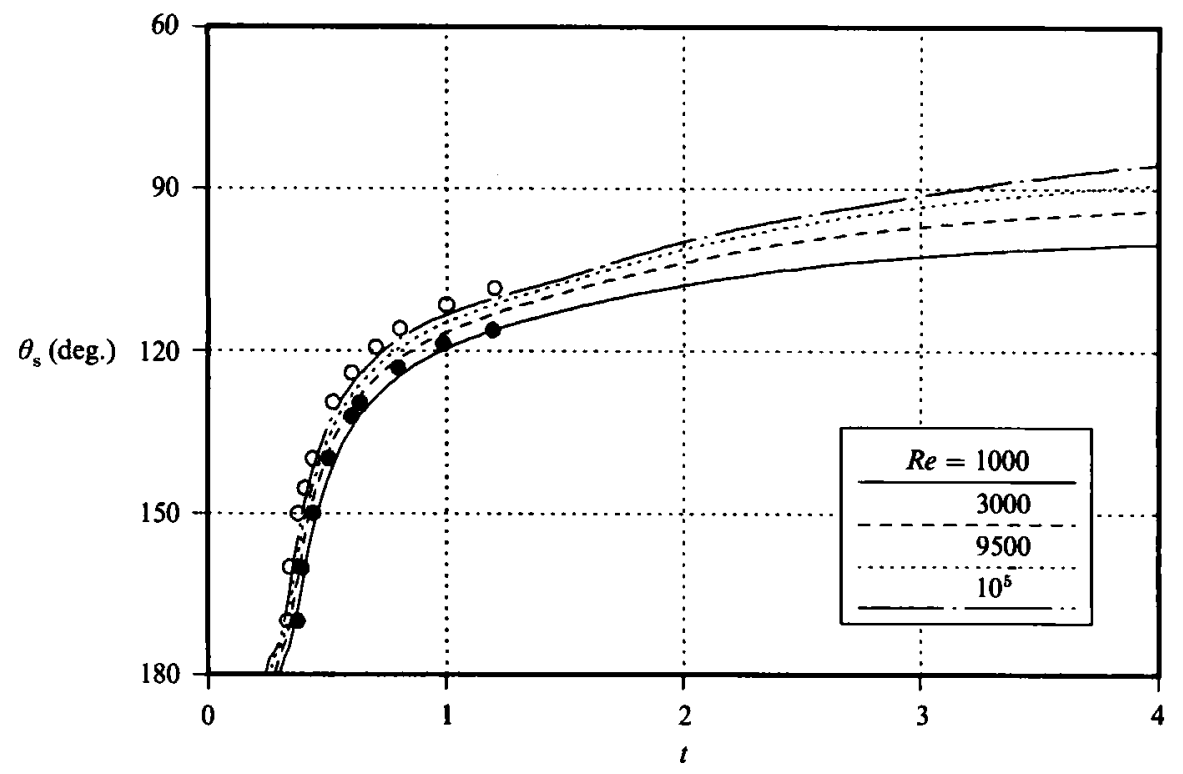

Figure 3. Time variation of the separation angle. Symbols are data from Collins \& Dennis $(1973 a): \mathcal{O}, R e=1000$ and $O, 10^{5}$.

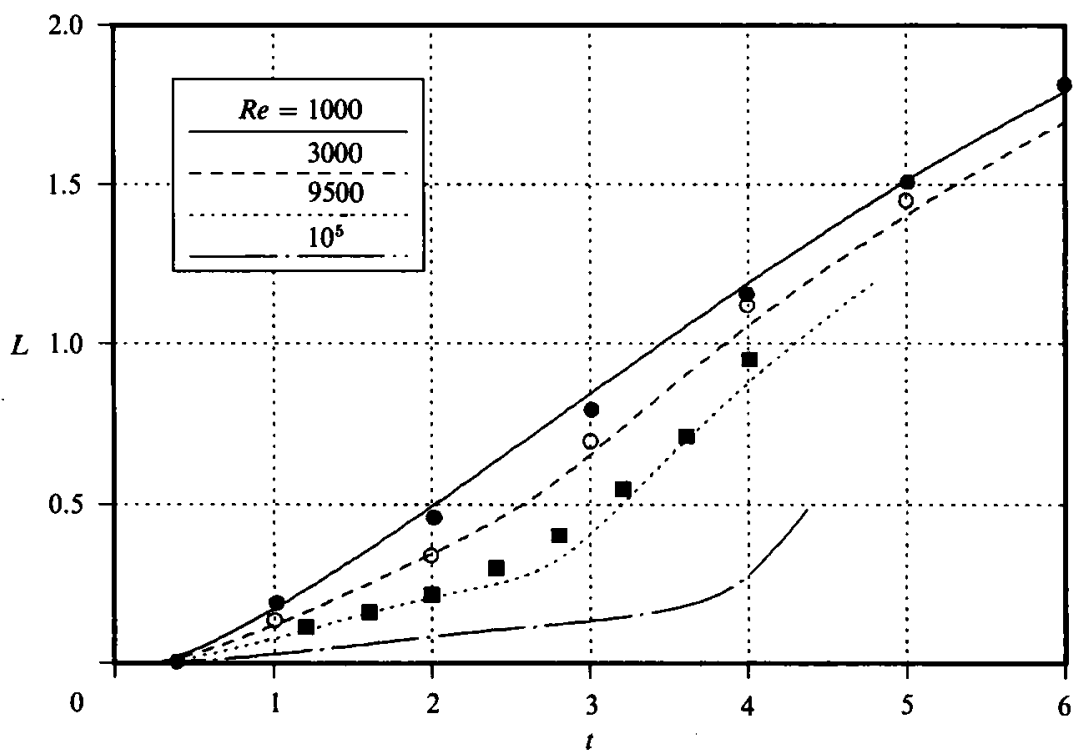

Figuke 4. Time variation of the wake length: $R e=1000$ from Ta Phuoc Loc (1980); O, $R e=3000 ; \square, R e=9500$ from Ta Phuoc \& Bouard (1985).

random walks to simulate the effect of viscous diffusion. The forces and flow patterns obtained by the present method are very regular and no smoothing technique for obtaining time or spatial averages is necessary. Nevertheless, the manner in which the vortices are convected and the circulations are redistributed is exactly the same as in a vortex-in-cell method for inviscid flows. Note also that the area-weighting scheme which locally smooths the vorticity field is indeed a mild source of numerical diffusion, even though its effect becomes smaller as the meshing is finer. It is therefore in this sense that we do not claim that the method, at least in its present form, is suitable for simulating turbulent flows unless there is enough grid resolution. 

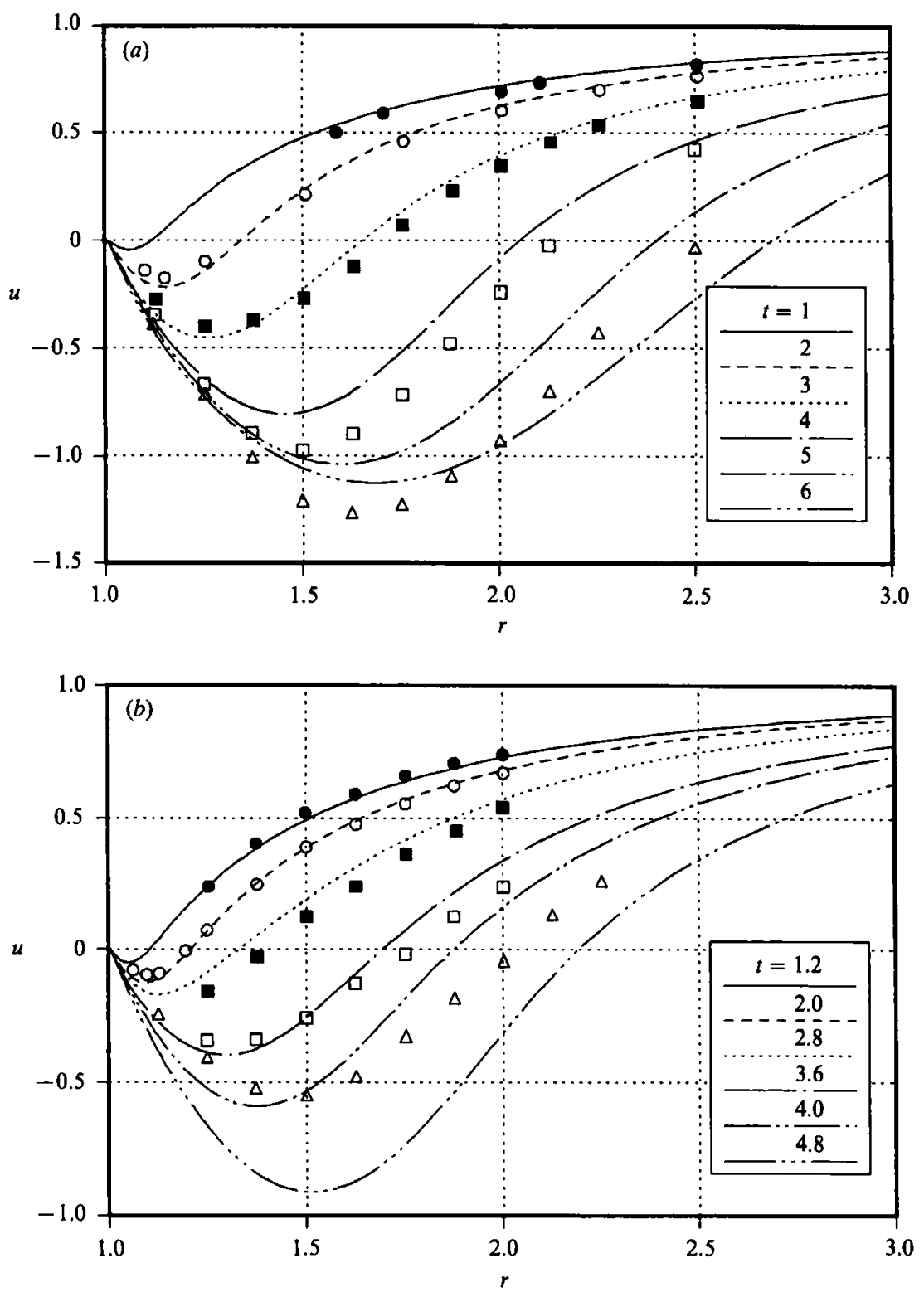

Figure 5. Comparison of radial velocities on the symmetric axis behind the cylinder for $(a)$ $R e=3000 ; \bigcirc, t=1 ; \bigcirc, 2 ; \square, 3 ; \square, 4, \triangle, 5 ;(b) R e=9500: \bigcirc, t=1.2 ; 0,2.0 ; \square, 2.8 ; \square, 3.6$; $\triangle$, 4.0. Discrete data are taken from Bouard \& Coutanceau (1980).

To ensure the validity of the present study, the flow patterns are restricted only to initial stages of flow establishment (especially at high Reynolds numbers) at which the real flow is most likely to be laminar.

\section{Numerical results and discussion}

The domain of ealculations is limited to the upper half-plane because of the flow symmetry in the early stages of development. The flows considered are of Reynolds numbers $R e=300,550,10^{3}, 3000,9500,20000,10^{5}$ and $10^{6}$. Numerical results are 

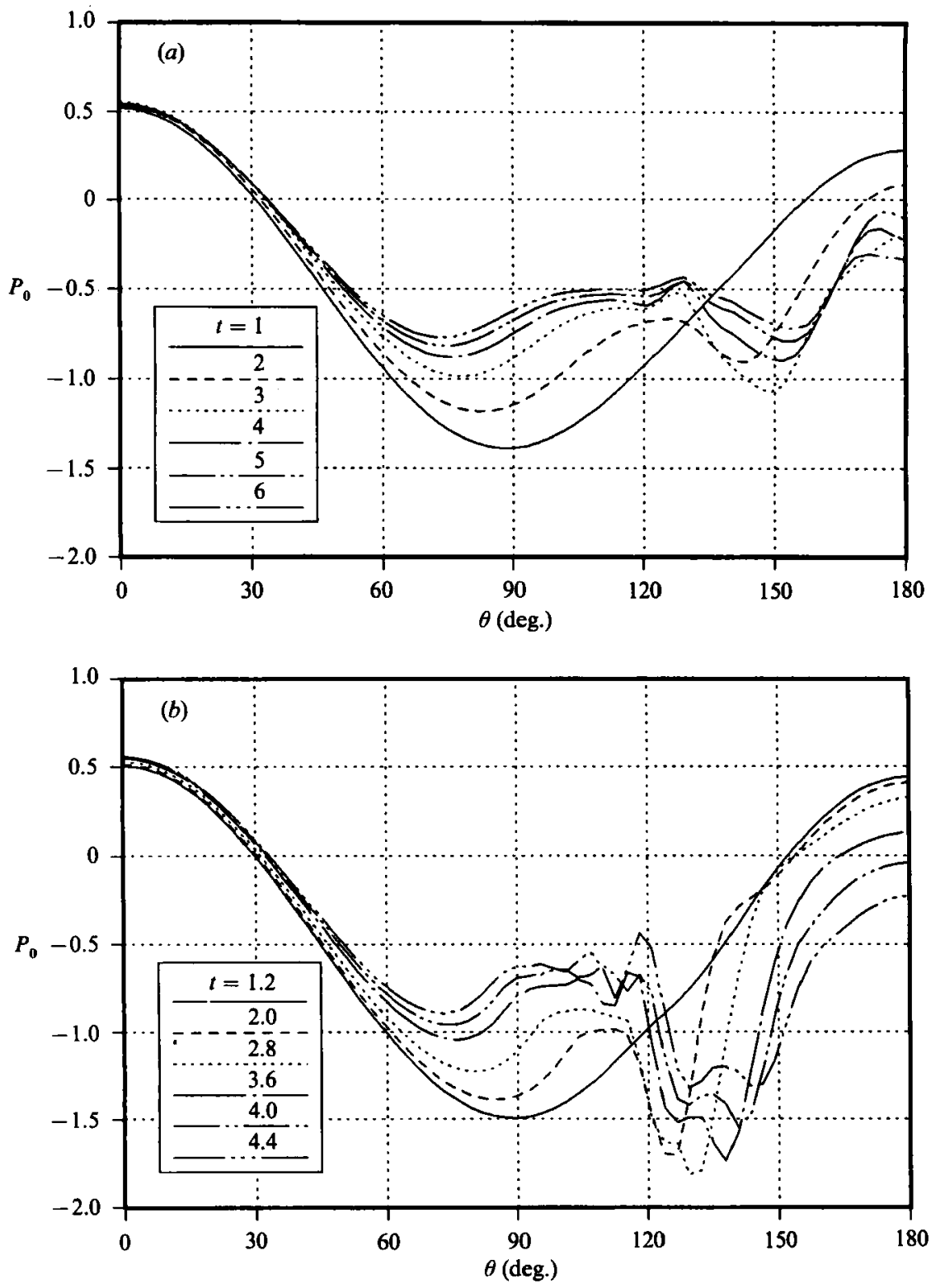

Figure 6. Time variation of the surface pressure for $(a) R e=3000,(b) 10^{5}$.

presented for time variations of the separation angles (figure 3), wake lengths (figure 4), velocities on the axis of symmetry in the near wake (figure 5), surface pressures (figure 6), drag coefficients (figure 7), surface vorticity distributions (figure 8) and streamline patterns (figures 9,10,14-17). In figures 11-13 and 18, some typical streamline patterns from the computed results and the results of flow visualization experiments are compared. The basic grid is $p \times q=128 \times 200$; the value of $r_{0}$ is 25 in all cases while the surface radial mesh spacing is taken to be the standard deviation of diffusion over one time step for each Reynolds number. The grid dependence has been checked by changing the value of $r_{0}$ and by multiplying the standard deviation by a factor close to one; no substantial difference was found. Nevertheless, the effects of the time step $\Delta t$ and the azimuthal size $\Delta \theta$ were analysed in particular for 

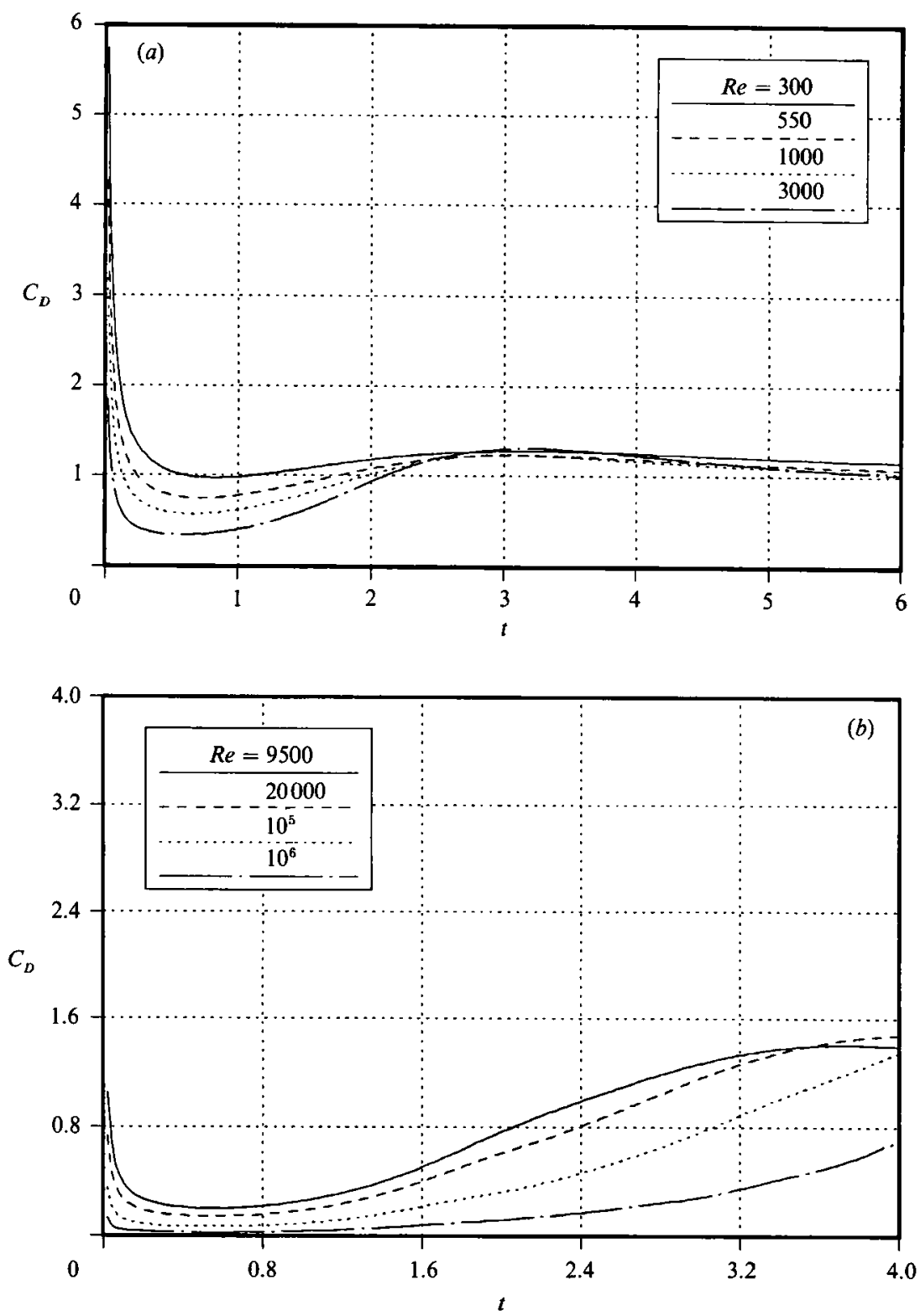

Figure $7(a, b)$. For caption see facing page.

$R e=3000$ and 9500 ; these cases have been carefully investigated by flow visualization techniques. Comparisons presented below show that the grid $p \times q=128 \times 200$ with the time step $\Delta t=0.02$ for all the cases under consideration is satisfactory. All the calculations are performed on a Convex C1/XP Machine; each cycle from (i) to (iv) takes nearly $1.25 \mathrm{CPU}$ seconds.

$$
\text { 4.1. } R e=300,550,1000,3000
$$

Figure 3 shows the time evolution of the separation angles $\left(\theta_{\mathrm{S}}\right)$. Flow separation occurs earlier as the Reynolds number is increased. For each Reynolds number, the separation angle decreases rapidly and tends toward a stationary value, which is, 

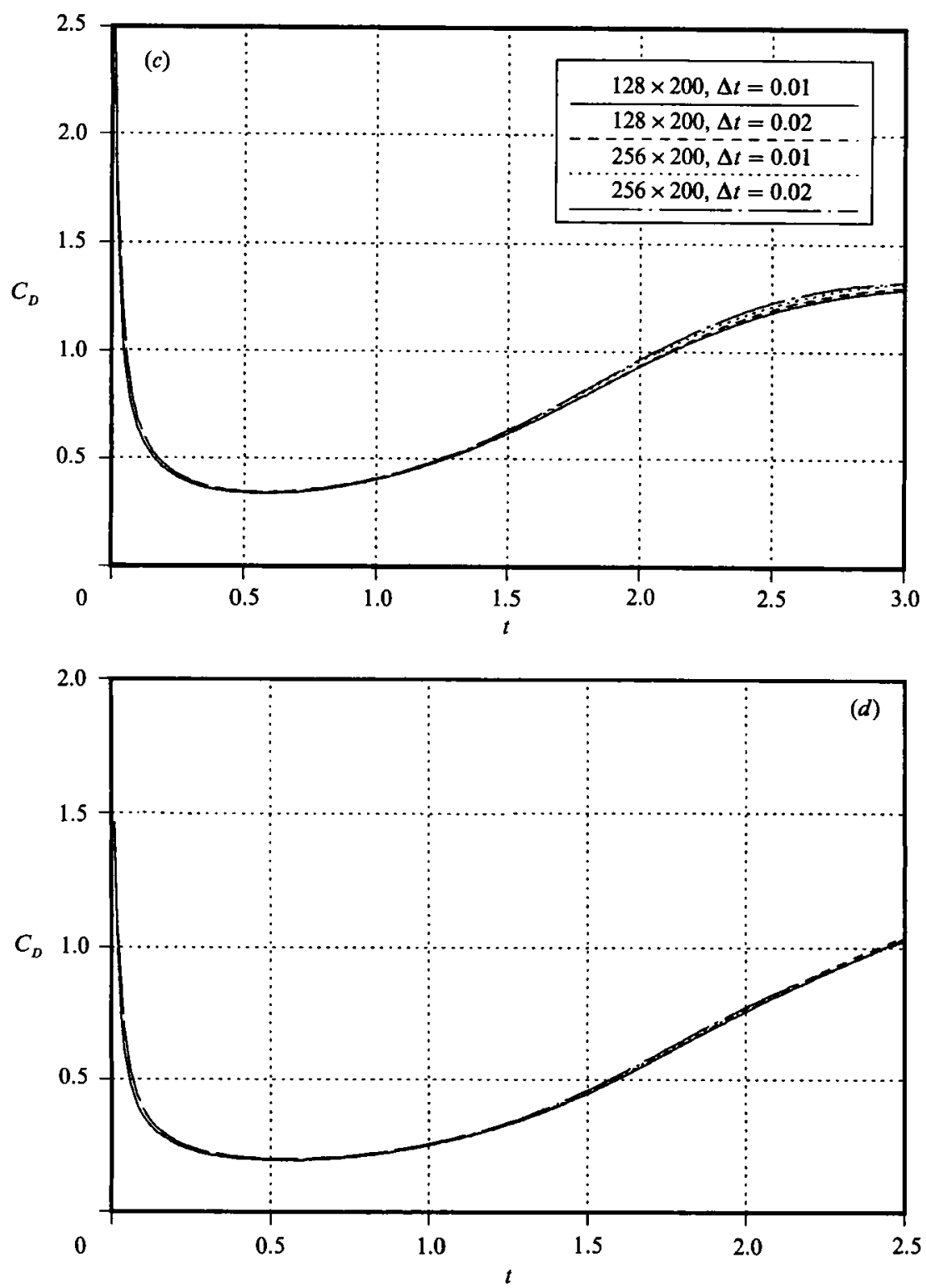

Figure 7. Time evolution of the drag coefficient: $(a, b)$ at various Reynolds numbers; $(c, d)$ with different timesteps and grids for $(c) R e=3000$ and $(d) 9500$.

however, greater than $90^{\circ}$ up to $t=6$. The result for $R e=1000$ shows a very close agreement with that of Collins \& Dennis (1973a) up to $t=1.2$. Figure 4 shows that the wake lengths $\left(L=L^{*} / a\right)$ for $R e=1000$ and 3000 increase almost linearly since their appearance. ( $L^{*}$ denotes the dimensional wake length.) They are found to be in good agreement with the numerical results of Ta Phuoc Loc (1980). Figure 5(a) shows a comparison of some radial velocities $(u)$ on the symmetric axis behind the cylinder with those obtained by Bouard \& Coutanceau (1980) for $R e=3000$. The agreement is quite good up to $t=3$. It is seen that the recirculation is strongest in the middle wake along the axis of symmetry. 

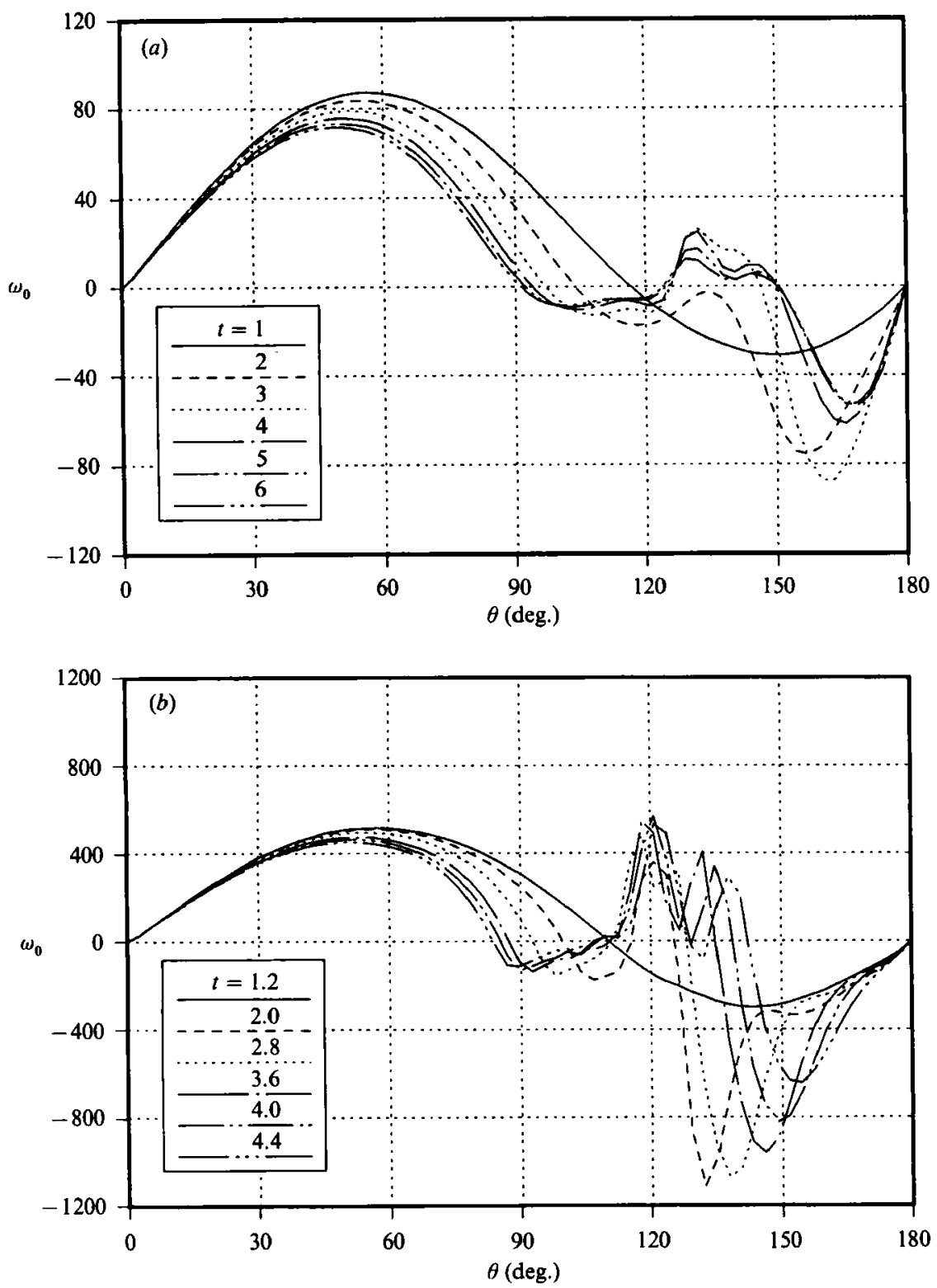

FigURE 8. Time variation of the surface vorticity for $(a) R e=3000,(b) 10^{5}$.

The surface pressure $\left(P_{0}\right)$ presented in figure $6(a)$ looks much like that at $R e=550$ in Collins \& Dennis $(\mathbf{1 9 7 3 b})$ at relatively small times. It is seen that the pressure recovers with more difficulty at lower Reynolds numbers; the surface pressure distribution has the tendency to be stationary as time is increasing. This tendency is also observed in the surface vorticity $\left(\omega_{0}\right)$ distribution, and seems to be stronger as the Reynolds number is decreased.

These facts indicate that the drag force may also tend to be stationary. This is indeed the case, as reflected by the drag coefficients $\left(C_{D}\right)$ shown in figure $7(a)$. The drag coefficient typically falls rapidly in the beginning to a minimal value, then increases smoothly to a mildly changing value. It is seen that at $t=6$ the drag 

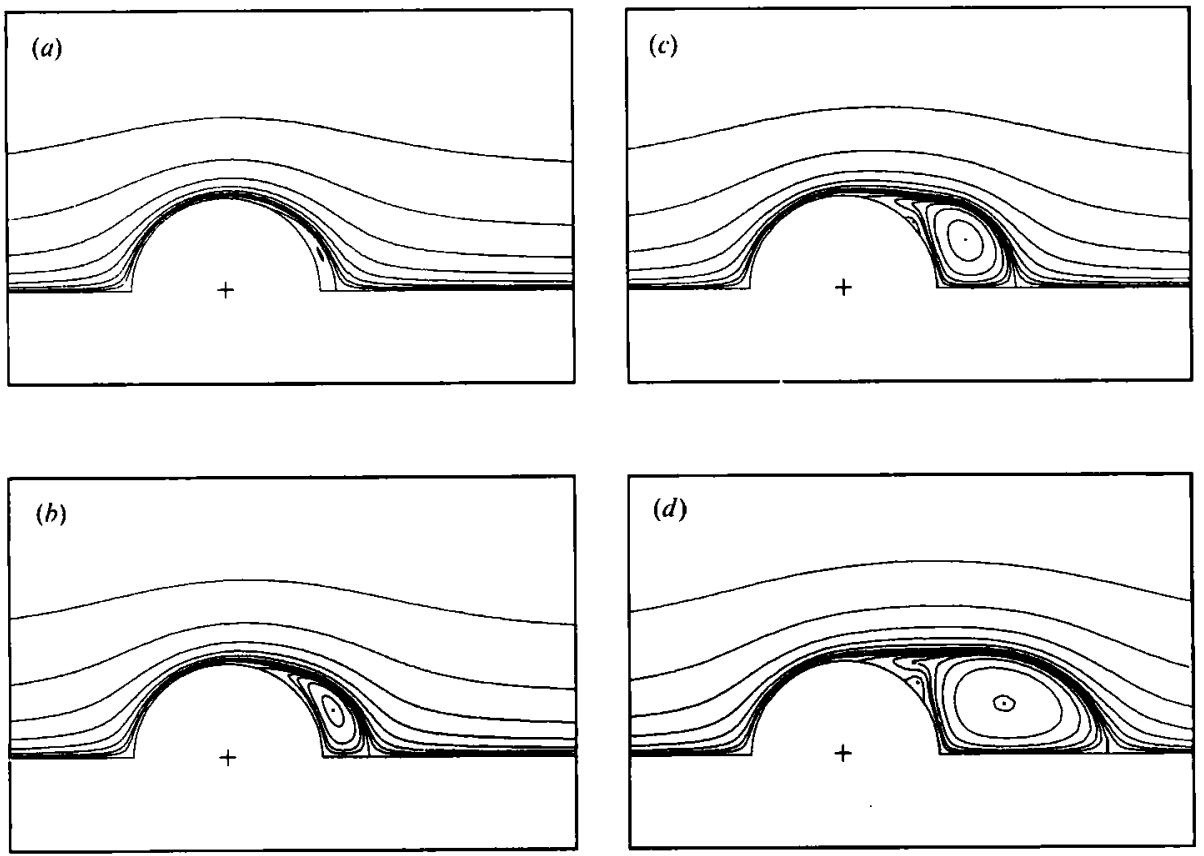

FIGURE 9. Instantaneous streamline patterns for $R e=1000$ at various times: (a) $t=1,(b) 2,(c) 3,(d) 6$.
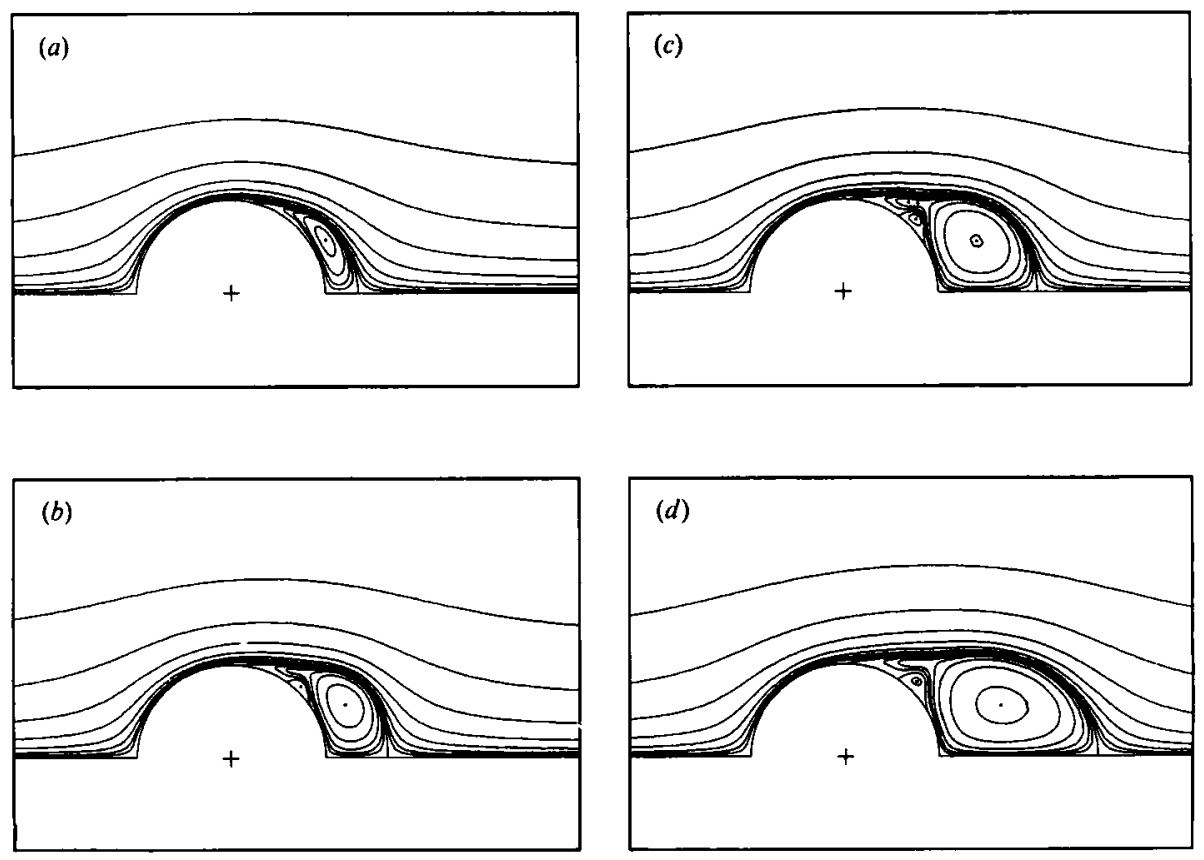

FIgURE $10(a-d)$. Instantaneous streamline patterns for $R e=3000$ at various times: (a) $t=2,(b) 3,(c) 4,(d) 6$.

coefficients are close to one; a lower value corresponds to a higher Reynolds number. This situation appears to be consistent with the stationary drag curve for a cylinder in the range under consideration; see e.g. Panton $(1984, \mathrm{p} .387)$. The effects of $\Delta \theta$ and the time step $\Delta t$ are analysed in figure $7(c)$. It is found that the drag force is almost 
(a)

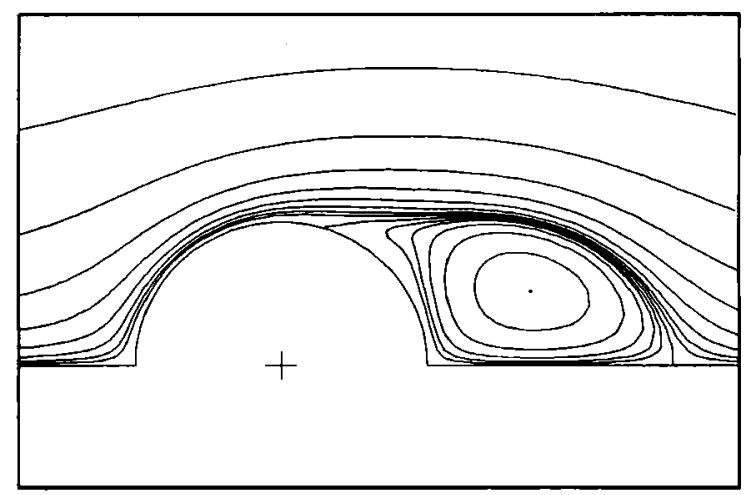

(b)

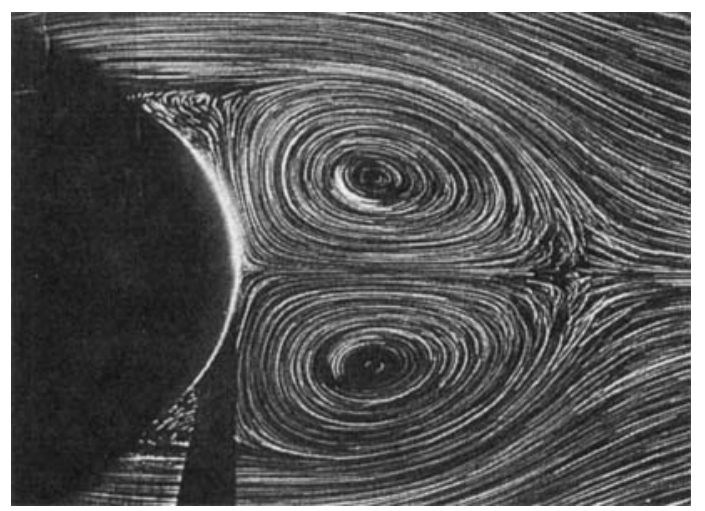

Figure 11. Comparison of the streamline patterns for $R e=300$ at $t=5:(a)$ the present method, $(b)$ flow visualization by Bouard \& Coutanceau (1980).

insensitive to either of them. The computed drag curves coincide very well with each other during the whole period of calculation (even at small times). In other words, $\Delta t=0.02$ is an adequate time step for resolving the initial flow with the present hybrid vortex method along with (9) and (10) for the drag coefficients.

These facts imply that the vorticity distribution near and along the cylinder surface does not vary significantly with further decreasing $\Delta \theta$ or $\Delta t$. Figure $8(a)$ shows time variation of the surface vorticity distribution for $R e=3000$. The variation is generally mild; however, after about $t=2.3$ the surface vorticity changes its sign three times along the cylinder surface, most likely corresponding to the formation of a secondary vortex. Indeed, it is observed that the bulge phenomenon is typical prior to the formation of an isolated secondary vortex; see e.g. figures $9(b, c)$ and $10(a, b)$. The secondary vortex, being contained within the primary vortex, is near to the cylinder surface, and does not interact with the exter alal flow. This is consistent with the previous observations of Honji \& Taneda (1969) and Ta Phuoc Loc \& Bouard (1985). From figure 10(c), we observe one additional secondary vortex which is not next to the cylinder surface and thus not predictable from the surface vorticity. The two vortices can be thought to form the $\alpha$-phenomenon. Figures 11 and 12 show that the streamline patterns for $R e=300$ and 550 at $t=5$ compare very well with the flow visualization results of Bouard \& Coutanceau (1980). 
(a)

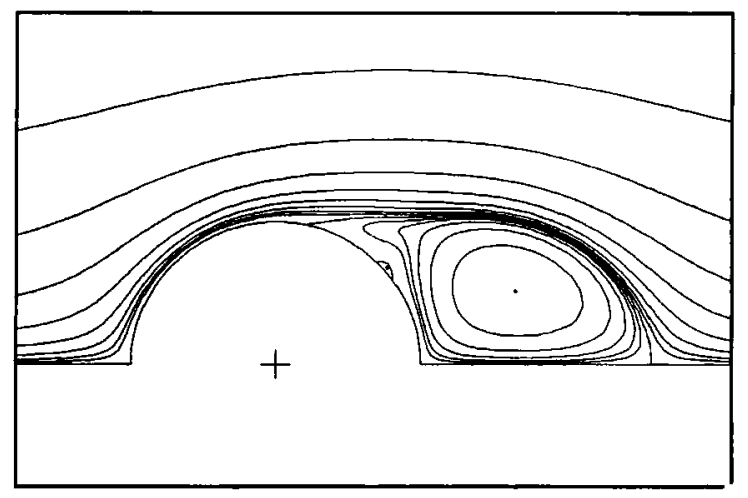

(b)

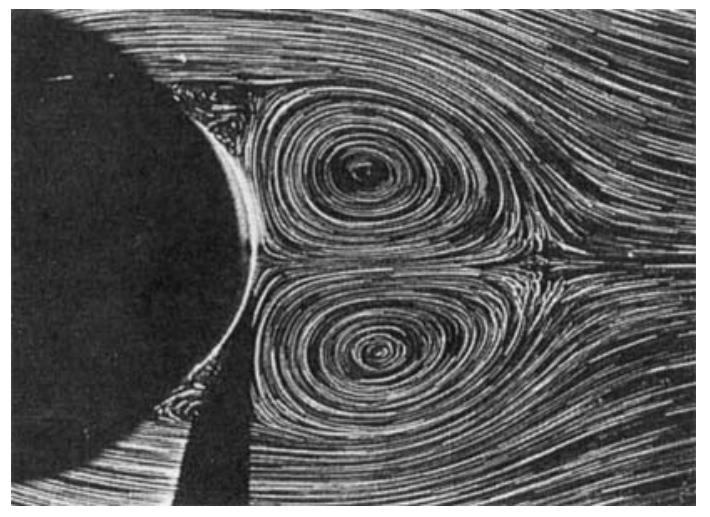

Figure 12. Comparison of the streamline patterns for $R e=550$ at $t=5:(a)$ present method, (b) flow visualization by Bouard \& Coutanceau (1980).

Furthermore, in figure 13, the grid dependence is checked against the time step $\Delta t$ and the azimuthal size $\Delta \theta$ for $R e=3000$ with $(p, q)=(128,200), \Delta t=0.02$ and $(p, q)=(256,200), \Delta t=0.01$. In the same figure, comparisons are made of the streamline patterns between the present results and the finite-difference and flow visualization results of Ta Phuoc Loc \& Bouard (1985). The grid $128 \times 200$ with $\Delta t=0.02$ is satisfactory in the comparisons of global streamline patterns, though the secondary vortices are slightly larger for the finer grid.

$$
\text { 4.2. } R e=9500,20000,10^{5}, 10^{6}
$$

Figure 3 shows that the separation angles decrease eventaully to values somewhat below $90^{\circ}$; the values differ little from each other. The result for $R e=10^{5}$ is found to be in close agreement with that of Collins \& Dennis $(1973 a$ ). Figures 4 and $5(b)$ (for $R e=9500$ ) show comparisons of the wake length and some radial velocities on the axis of symmetry behind the cylinder. The general agreement is quite good, although the discrepancy with the experimental results of Bouard \& Coutanceau (1980) increases from $t=2.8$. Figures $6(b)$ and $8(b)$ (for $R e=10^{5}$ ) indicate that in these flows, the surface pressure and vorticity change violently over the cylinder surface; two or more vortices near the surface are therefore likely. It is noted that for $R e=10^{5}$ 
(a)

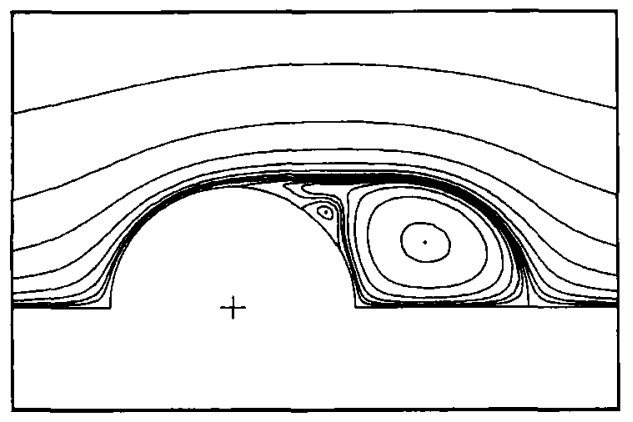

(b)

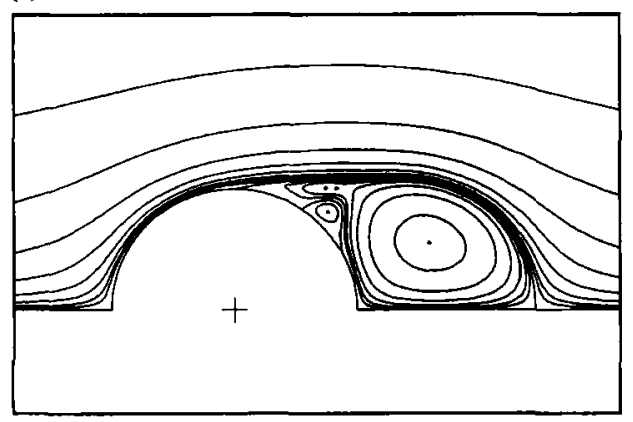

(c)

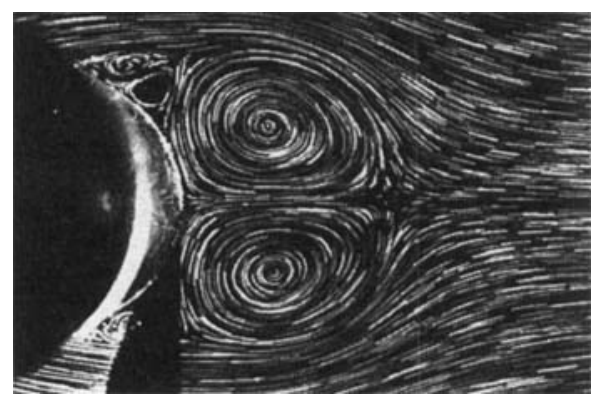

(d)

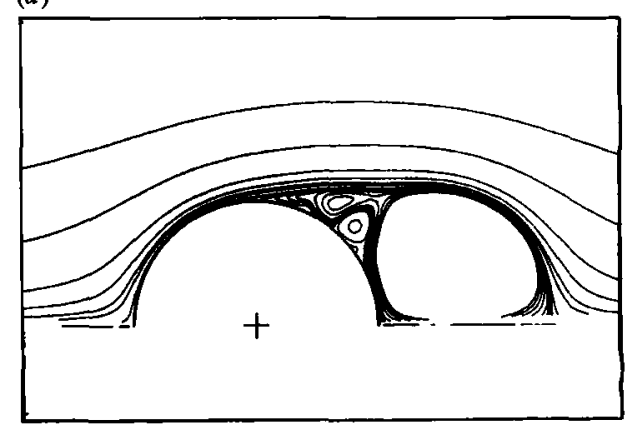

Figure 13. Comparison of the streamline patterns for $R e=3000$ at $t=5:(a)$ present method, $p \times q=128 \times 200, \Delta t=0.025 ;(b)$ present method, $p \times q=256 \times 200, \Delta t=0.01 ;(c)$ flow visualization by Ta Phuoc Loc \& Bouard (1985); (d) finite-difference method by Ta Phuoc Loc \& Bouard (1985).

the vorticity may exceed 1000 while for $R e=10^{6}$ the value may even exceed 3500 . In these cases, we observe again that the bulge phenomenon is typical prior to the formation of an isolated secondary vortex. Furthermore, it is found that the $\alpha$-and $\beta$-phenomena are typical in the early phases of flow establishment. The $\alpha$ phenomenon can be seen from, for example, figures $14(c, d), 15(d), 16(d)$, while the $\beta$-phenomenon can be seen from, for example, figures $14(a, b), 15(a, b), 16(a-c)$, and $17(a-d)$. In all the cases, the wake length increases linearly initially and then increases with a faster rate. The faster rate corresponds to the forewake ( $\beta$ phenomenon) soon developing into the main wake (cf. figure 4).

Figure $7(b)$ shows time histories of the drag coefficients. The typical pattern of the drag curves is basically the same as that at the lower Reynolds numbers except that for $R e=10^{6}$. It is interesting that the drag coefficient for $R e=10^{6}$ is relatively quite small for an initial portion of time; the coefficient may even be nearly zero. This may possibly correspond to the experimental result that the (stationary) drag coefficient drops rapidly at $R e \approx 3 \times 10^{5}$ (cf. Tritton $1988, \mathrm{p} .34$ ). The effects of the size $\Delta \theta$ and the time step $\Delta t$ on the drag coefficient are analysed in figure $7(d)$ for $R e=9500$. It is found again that the computed drag curves coincide very well with each other. These observations more or less confirm that the present method does indeed provide some useful and reliable information at high Reynolds numbers. Owing to the rapid 

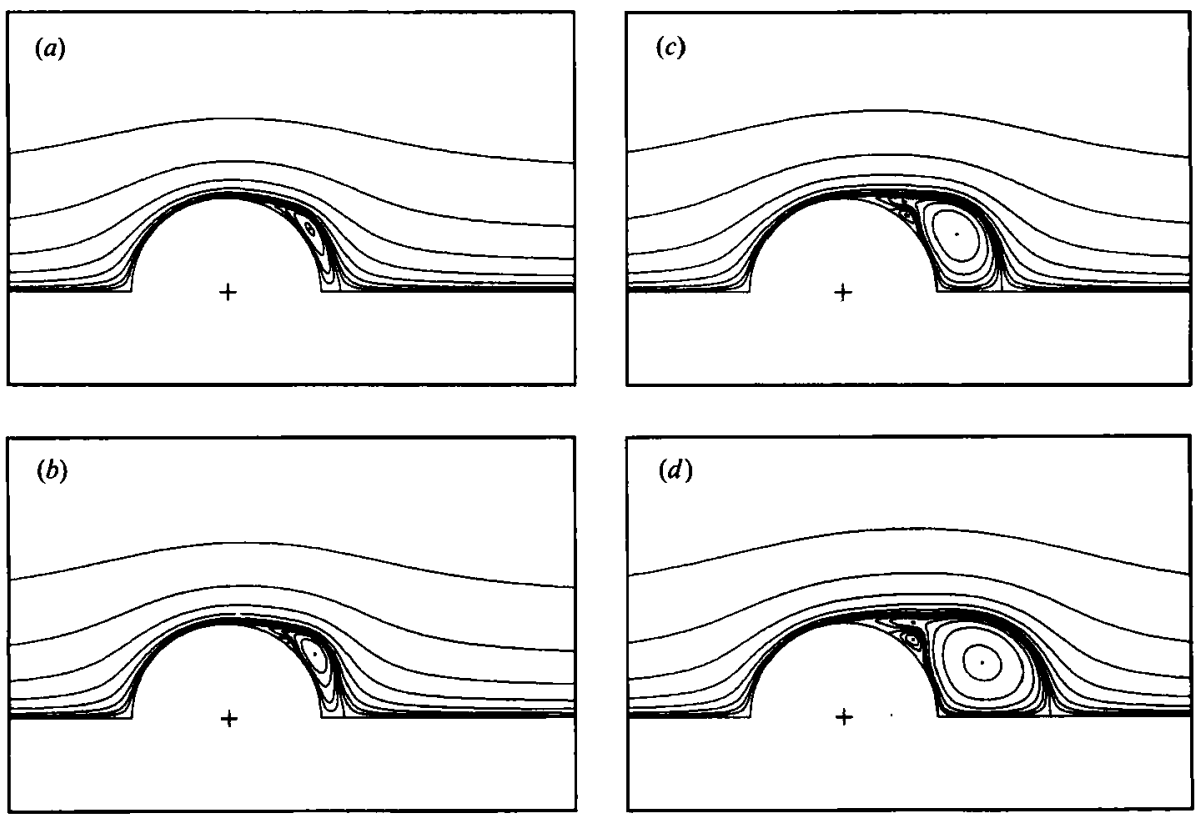

FiguRE 14. Instantaneous streamline patterns for $R e=9500$ at various times:

(a) $t=2.0,(b) 2.4,(c) 3.6,(d) 4.8$.
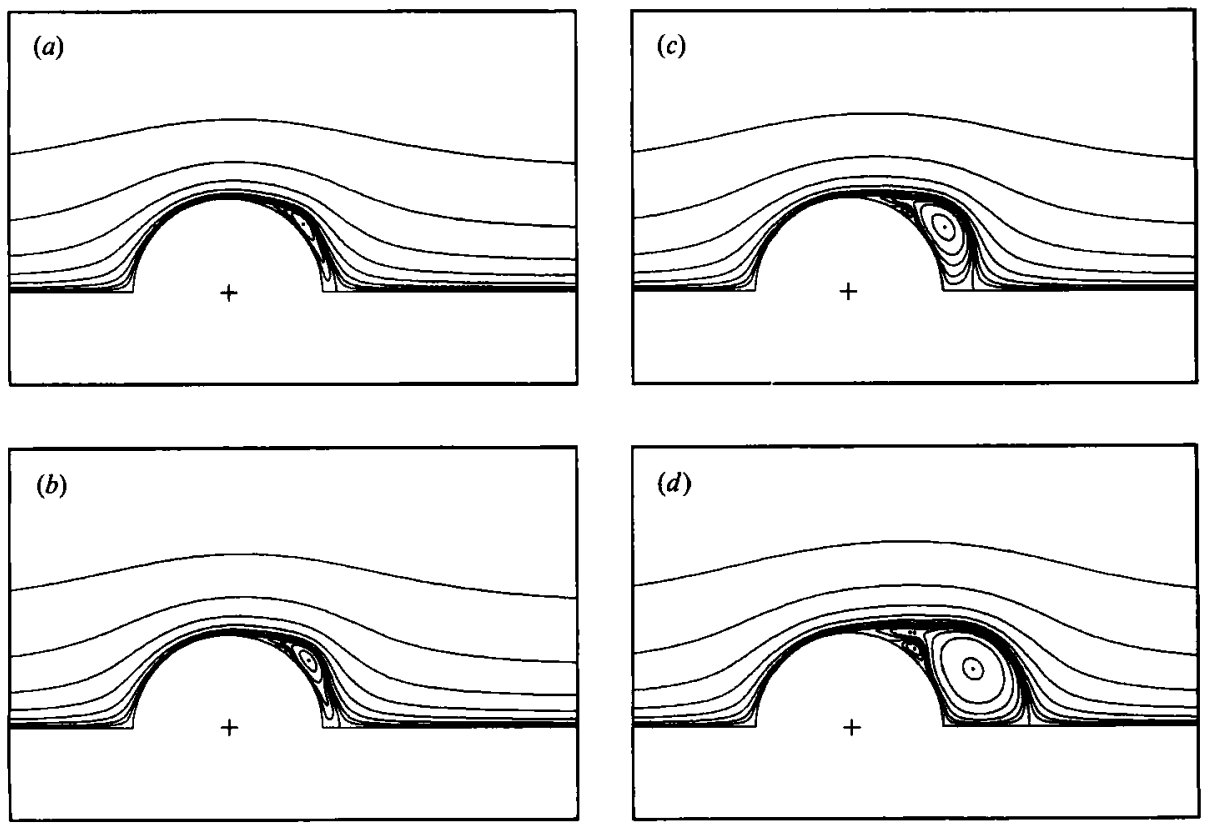

FIGURE 15. Instantaneous streamline patterns for $R e=20000$ at various times: (a) $t=2.0,(b) 2.4,(c) 3.2,(d) 4.4$.

change of the flow at high Reynolds numbers, it should not be a surprise that there is an increasing discrepancy between the present results and previous numerical results of Ta Phuoc Loc \& Bouard (1985) and experimental results of Bouard \& Coutanceau (1980). Ta Phuoc Loc \& Bouard used steady-state solutions of low- 

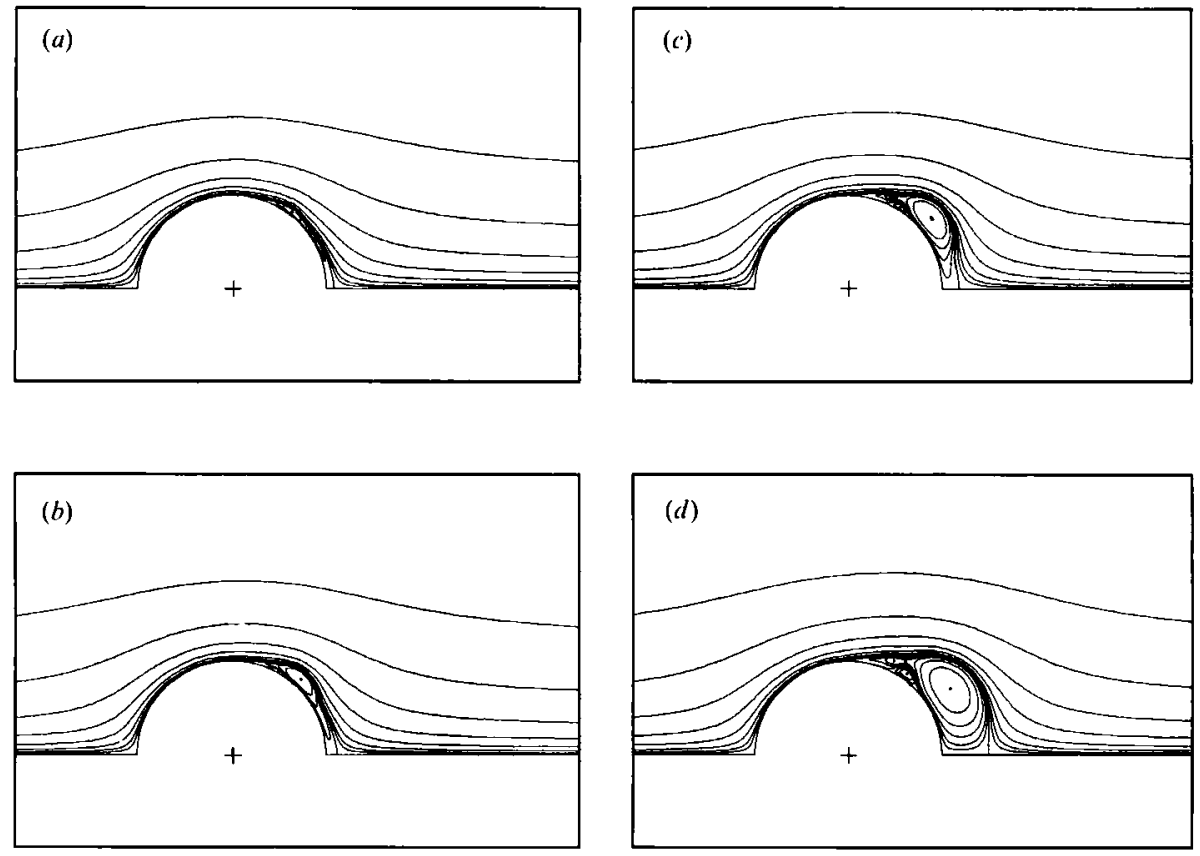

FIGURE 16. Instantaneous streamline patterns for $R e=10^{5}$ at various times: (a) $t=2.0,(b) 2.8,(c) 3.6,(d) 4.4$.
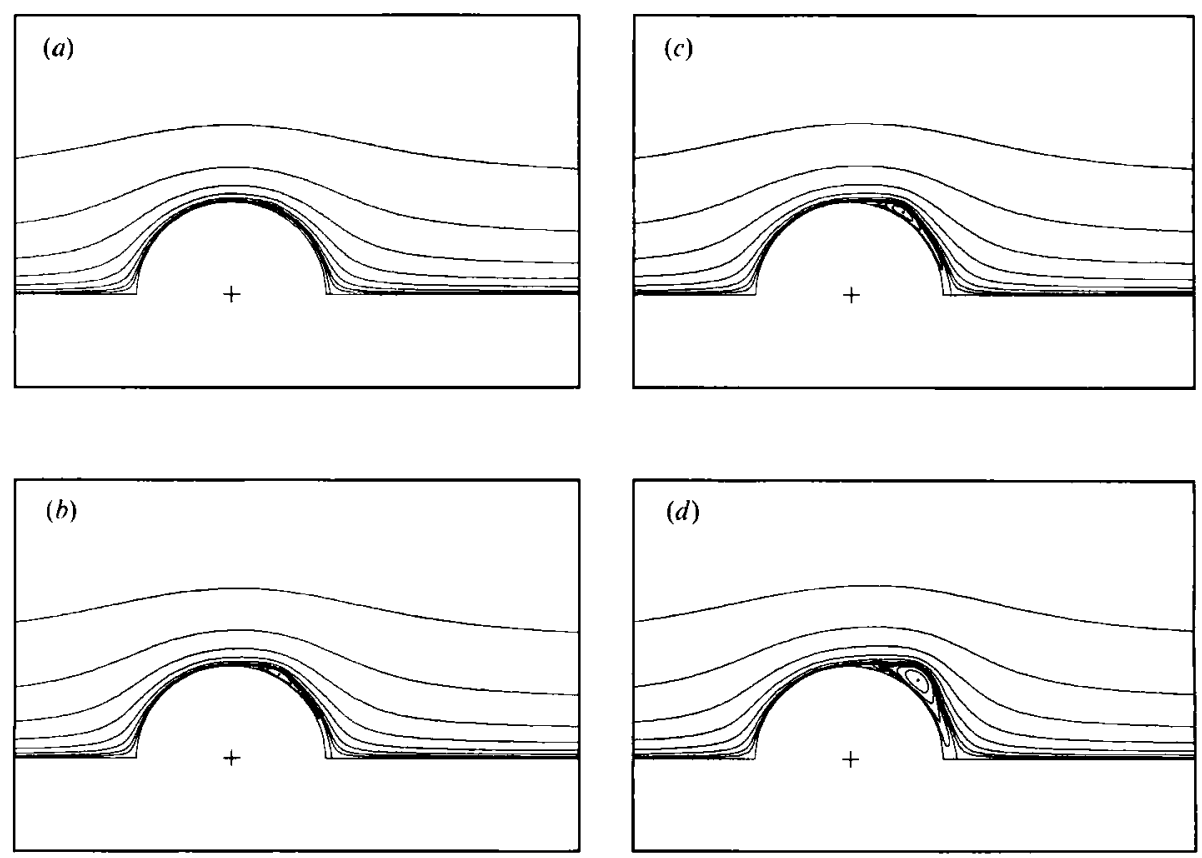

Figure 17. Instantaneous streamline patterns for $R e=10^{6}$ at various times: (a) $t=2.4,(b) 3.2,(c) 3.6,(d) 4.4$.

Reynolds-number flows as initial conditions to obtain good agreement between the numerical and flow visualization experiments. But there is also the possibility that this discrepancy is due to the finite difference's suffering from numerical diffusivity or that the real flow hardly remains two-dimensional and becomes eventually 

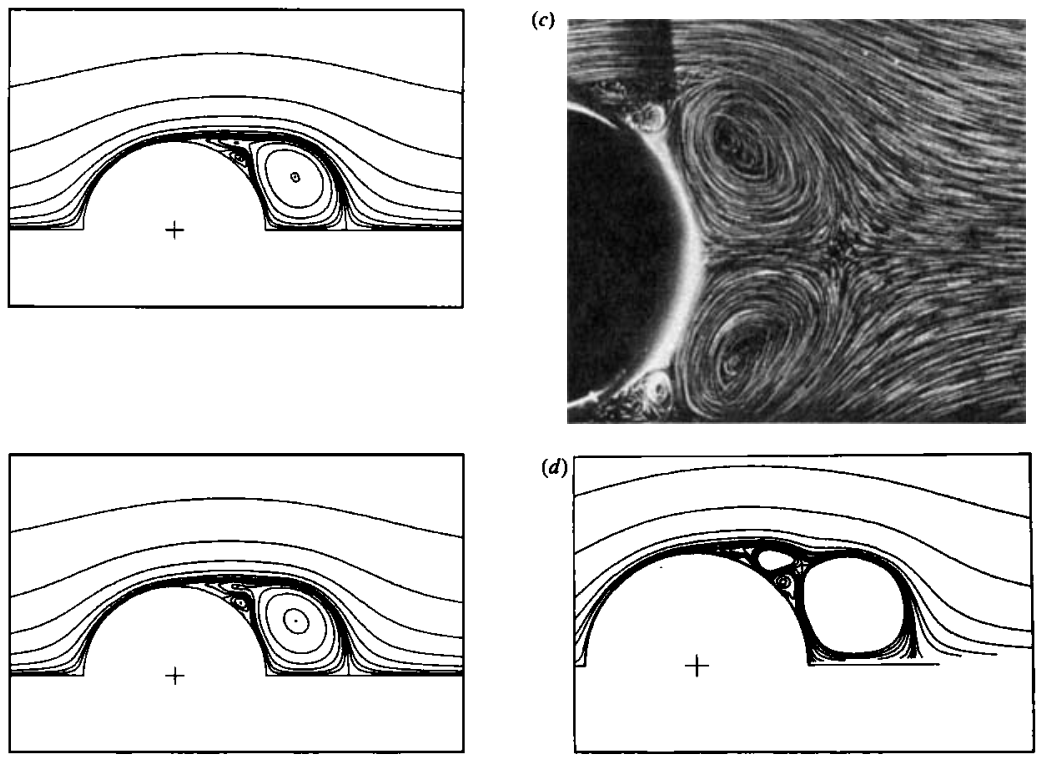

Figure 18. Comparison of the streamline patterns for $R e=9500$ at $t=4.0:(a)$ present method, $p \times q=128 \times 200, \Delta t=0.02 ;(b)$ present method, $p \times q=256 \times 200, \Delta t=0.01 ;(c)$ flow visualization by Ta Phuoc Loc \& Bouard (1985); (d) finite-difference method by Ta Phuoc Loc \& Bouard (1985).

turbulent at high Reynolds numbers. However, this should be remedied in threedimensional calculations. Nevertheless, we have extended the present work to flows of Reynolds numbers up to $\mathbf{1 0}^{6}$, and still obtained very stable numerical results, which indicates that for the present method, two-dimensional disturbances do not lead to divergence of solutions over a long period of calculation. In figure 18, the grid dependence is checked against the time step $\Delta t$ and the azimuthal size $\Delta \theta$ for $R e=9500$ with $(p, q)=(128,200), \Delta t=0.02$ and $(p, q)=(256,200), \Delta t=0.01$. In the same figure, comparisons are also made of between the streamline patterns from the present results and the finite-difference and flow visualization results of Ta Phuoc Loc \& Bouard (1985). The grid $128 \times 200$ with $\Delta t=0.02$ is found to be satisfactory in comparisons of the global streamline patterns. However, the calculation based on the finer grid indicates clearly a small but visible tertiary vortex next to the cylinder surface; this tertiary vortex is not resolved on the coarser grid.

\section{Concluding remarks}

We have shown how a hybrid vortex algorithm can be implemented in a deterministic way; the method is highly stable and reliable even for flows at moderately high Reynolds numbers. The numerical results compare very well with previous analytic, finite-difference and experimental results. The method can also be readily applied to investigate various other physical problems. Some research subjects related to the present study, including the effect of rotation and flow around geometrically non-smooth surfaces, are currently under investigation; the results will be reported elsewhere. Finally, it is noted that the authors were informed by one of the referees that Graham (1988) has also taken up another (different) deterministic approach to the vortex (particle) method. Graham's work contains a brief discussion of the conservation of the moments of the vorticity field. It is thus helpful to include 
this reference here although a comparative study between the two methods is apparently unavailable at present.

The authors thank all the referees for their helpful comments. This work is partially supported by the National Science Council of R.O.C. under Contract No. NSC79-0401-E002-25.

\section{REFERENCES}

Bar-Lev, M. \& Yana, H. T. 1975 Initial flow field over an impulsively started circular cylinder. J. Fluid Mech. 72, 625-647.

Beale, J. T. \& MAJDA, A. 1985 High order accurate vortex methods with explicit kernels. J. Comput. Phys. 58, 188-208.

Bouard, R. \& Coutanceav, M. 1980 The early stage of development of the wake behind an impulsively started circular cylinder for $40<R e<10^{4}$. J. Fluid Mech. 101, 583-607.

Cheer, A. Y. 1983 A study of incompressible 2-D vortex flow past a circular cylinder. SIAM J. Sci. Stat. Comput. 4, 685-705.

CHEer, A. Y. 1989 Unsteady separated wake behind an impulsively started cylinder in slightly viscous fluid. J. Fluid Mech. 201, 485-505.

Choris, A. J. 1973 Numerical study of slightly viscous flow. J. Fluid Mech. 57, 785-796.

Chorin, A.J. 1978 Vortex sheet approximation of boundary layers. J. Comput. Phys. 27, 428-442.

Christiansen, J.P. 1973 Numerical simulation of hydrodynamics by the method of point vortices. J. Comput. Phys. 13, 363-379.

Collins, W. M. \& Dennis, S. C. R. $1973 a$ The initial flow past an impulsively started circular cylinder. Q.J. Mech. Appl. Maths 26, 53-72.

Collins, W. M. \& Dennis, S. C. R. $1973 b$ Flow past an impulsively started circular cylinder. J. Fluid Mech. 60, 105-127.

Ghoniem, A. F., Chorin, A. J. \& Oppenheim, A. K. 1982 Numerical modeling of turbulent flow in a combustion tunnel. Phil. Trans. R. Soc. Lond. A 304, 303-325.

Graham, J.M. R. 1988 Computation of viscous separated flow using a particle method. In Numerical Methods for Fluid Dynamics, vol. 3, (ed. K. W. Morton \& M. J. Baines), pp. 310-317. Oxford University Press.

HALD, O. H. 1979 The convergence of vortex methods II. SIAM J. Numer. Anal. 16, 726-755.

Honsi, H. \& TAneda, S. 1969 Unsteady flow past a circular cylinder. J. Phys. Soc. Japan 27, 1668-1677.

Leonard, A. 1980 Vortex methods for flow simulation. J. Comput. Phys. 37, 289-335.

LeOnaRD, A. 1985 Computing three-dimensional incompressible flows with vortex elements. Ann. Rev. Fluid Mech. 17, 523-559.

Panton, R. L. 1984 Incompressible Flow. Wiley.

SarpKaya, T. 1989 Computational methods with vortices - the 1988 Freeman Scholar Lecture. Trans. ASME T: J. Fluids Engng 111, 5-52.

Sethian, J. A. 1984 Turbulent combustion in open and closed vessels. J. Comput. Phys. 54, 425-456.

Smith, P. A. \& Stansby, P. K. 1988 Impulsively started flow around a circular cylinder by the vortex method. J. Fluid Mech. 194, 45-77.

TA Phuoc Loc 1980 Numerical analysis of unsteady secondary vortices generated by an impulsively started circular cylinder. $J$. Fluid Mech. 100, 111-128.

TA Phuoc Loc \& Bouard, R. 1985 Numerical solution of the early stage of the unsteady viscous flow around a circular cylinder: a comparison with experimental visualization and measurements. J. Fluid Mech. 160, 93-117.

Thoman, D. C. \& Szewczyк, A. A. 1969 Time-dependent viscous flow over a circular cylinder. Phys. Fluids Suppl. 12 II, 76-86. 
Tiemroth, E. C. 1986 Simulation of the viscous flow around a cylinder by the random vortex method. Ph.D. thesis, University of California, Berkeley.

Tritton, D. J. 1988 Physical Fluid Dynamics (2nd Edn). Oxford University Press.

WANG, C.Y. 1967 The flow past a circular cylinder which is started impulsively from rest. J. Maths \& Phys. 46, 195-202. 